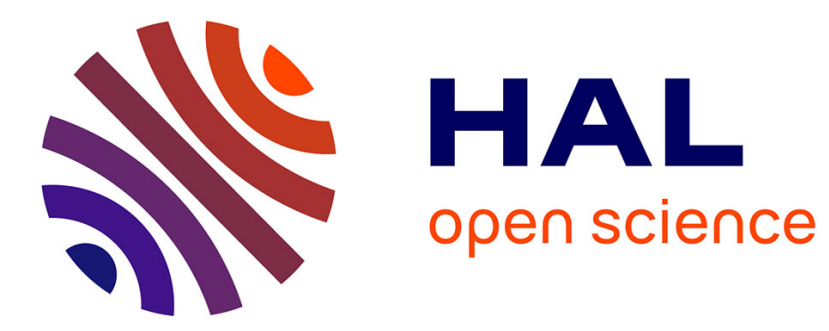

\title{
Flux jumps in hard superconductors in decreasing magnetic field
}

J. Sosnowski

\section{To cite this version:}

J. Sosnowski. Flux jumps in hard superconductors in decreasing magnetic field. Revue de Physique Appliquée, 1985, 20 (4), pp.221-224. 10.1051/rphysap:01985002004022100 . jpa-00245326

\section{HAL Id: jpa-00245326 https://hal.science/jpa-00245326}

Submitted on 1 Jan 1985

HAL is a multi-disciplinary open access archive for the deposit and dissemination of scientific research documents, whether they are published or not. The documents may come from teaching and research institutions in France or abroad, or from public or private research centers.
L'archive ouverte pluridisciplinaire HAL, est destinée au dépôt et à la diffusion de documents scientifiques de niveau recherche, publiés ou non, émanant des établissements d'enseignement et de recherche français ou étrangers, des laboratoires publics ou privés. 


\title{
REVUE DE PHYSIQUE APPLIQUÉE
}

\section{Flux jumps in hard superconductors in decreasing magnetic field}

\author{
J. Sosnowski \\ Dept. of Fundamental Research, Electrotechnical Institute, 04-703 Warsaw, Pożaryskiego 28, Poland
}

(Reçu le 18 avril 1984, révisé le 29 octobre, accepté le 11 janvier 1985)

\begin{abstract}
Résumé. - Les résultats de calcul des positions supérieures des sauts de flux dans des supraconducteurs de $2^{\mathrm{e}}$ espèce en champ magnétique décroissant sont présentés. Il est montré que les sauts de flux et les limites de stabilité correspondantes dépendent de la valeur maximale du champ magnétique pendant le cycle, ce qui influence principalement la première limite de stabilité en champ décroissant. Les positions des autres sauts de flux sont déterminées pour deux relations physiques simples entre la limite de stabilité et le champ aux sauts de flux. Le nombre maximal de sauts de flux et le champ à la limite de complète stabilité sont aussi discutés dans les deux cas.
\end{abstract}

\begin{abstract}
The results of calculations of higher flux jumps positions in hard superconductors in decreasing magnetic field have been presented. It has been shown that flux jumps and corresponding stability limits are dependent on maximal magnetic field value in the cycle, which influences mainly first stability limit in decreasing field. Positions of other flux jumps have been determined for two simple physical relations between stability limit and flux jumps field. Maximal number of flux jumps and full stability limit field have been in both cases also discussed.
\end{abstract}

\section{Introduction.}

In numerous papers devoted to the problem of instabilities and flux jumps (see for example [1, 2]) much attention was directed into the case of increasing magnetic field. Experimentally it was however frequently observed that numerous flux jumps appear also in decreasing magnetic field. From irreversible properties of hard superconductors follows also that occurrence of flux jumps cannot be described with the same conditions as given for increasing magnetic field, for example in [2]. Therefore an aim of the present paper is to consider conditions determining flux jumps positions in decreasing magnetic field. The case of first stability limit in decreasing magnetic field was investigated in a previous paper [3]. In present one we concentrate on an existence of higher flux jumps in decreasing from maximal value in cycle $B^{m}$ to null magnetic field.

Although the principles of arising instabilities in this case are the same as described in [1, 2] for increasing magnetic field, however from irreversible properties of hard type-II superconductors we receive for decreasing magnetic field modified form of equilibrium equation which leads to new stability limits and flux jumps positions.

\section{Method of calculations.}

Lorentz and pinning forces in Cartesian coordinate system with an external magnetic field parallel to $z$-axis are expressed by equation :

$$
F_{\mathrm{L}}=-\frac{1}{\mu_{0}} B \frac{\partial B}{\partial x}, \quad F_{\mathrm{p}}=\frac{\alpha B}{B+B^{0}} \operatorname{sgn} \frac{\partial B}{\partial x}
$$

where $\mu_{0}$ is magnetic permeability, $\boldsymbol{B}$ magnetic induction profile, while $\alpha$ and $B^{0}$ parameters determine pinning forces in Kim's critical state model. As it has been shown in [1] stability limit field is determined by nonvanishing solution of an equilibrium equation which defines a disturbance function $-\beta_{n}(x)$. We investigate the case of an existence manifold flux jumps then index $n$ numerates subsequent disturbance functions. For the case of decreasing magnetic field we search negative disturbance function $-\beta_{n}(x)$ $<0$, which induces the variations of Lorentz and pinning forces of an equal magnitude :

$$
\Delta F_{\mathrm{L}}+\Delta F_{\mathrm{p}}=\frac{1}{\mu_{0}} \frac{\partial\left(B \beta_{n}\right)}{\partial x}+\frac{\partial F_{\mathrm{p}}}{\partial T} \Delta T-\frac{\partial F_{\mathrm{p}}}{\partial B} \beta_{n}=0
$$


$\Delta T$ in equation (2) means temperature increase connected with dissipation of power $Q$ in the process of fluxoids movement against pinning forces and fluxoids annihilation caused by disturbance function $-\beta_{n}(x)$. This temperature increase may be simply estimated from Maxwell's equations :

$$
\begin{gathered}
\frac{\mathrm{d} E_{y}}{\mathrm{~d} x}=-\dot{B} \approx \frac{\beta_{n}}{\Delta t}, \quad \mu_{0} j_{y}=-\frac{\partial B}{\partial x}, \\
Q=E_{y} j_{y} \Delta t=c \Delta T
\end{gathered}
$$

where $\Delta t$ is small time period in which dissipation appears, while $c$ - means specific heat per unit volume. $\Delta T$ is the corresponding temperature increase. Integrating equation (3) and introducing next result into equation (2) we receive the conditions on the limited stability field in the form of nonlinear differentiallyintegral equation :

$\frac{1}{\mu_{0}} \frac{\partial\left(B \beta_{n}\right)}{\partial x}+\frac{1}{\mu_{0} c} \frac{\partial B}{\partial x} \frac{\partial F_{\mathrm{p}}}{\partial T} \int_{x}^{x_{n-1}} \beta_{n} \mathrm{~d} x-\frac{\partial F_{\mathrm{p}}}{\partial B} \beta_{n}=0$

where $x_{n-1}$ is the corresponding penetration depth.

\section{Results.}

Assuming as in [1] that value of parameter $B^{0}$ in equation (1) is temperature independent, on the basis of explicit form of Lorentz and pinning forces given by equation (1) we receive an constitutive equation describing $n$-th disturbance function for decreasing magnetic field :

$$
\frac{\left[B(x)+B^{0}\right]^{2}}{\mu_{0} \alpha} \frac{\mathrm{d} \beta_{n}}{\mathrm{~d} x}+\beta_{n}+\frac{1 \partial \alpha}{c \partial T} \int_{x}^{x_{n-1}} \beta_{n} \mathrm{~d} x=0 .
$$

It should be noted that applying equations (1)-(3) to the case of increasing magnetic field we receive an equation of the type equation (5) but with negative sign in second term, what leads to the results given in $[1,2]$.

The magnetic induction profile $B(x)$ for decreasing magnetic field $B_{\mathrm{e}}$ is described with an equation :

$$
\left[B(x)+B^{0}\right]^{2}=\left[B_{\mathrm{e}}+B^{0}\right]^{2}+2 \mu_{0} \alpha x .
$$

Differentiating equation (5) we receive following second order equation which can be solved analytically :

$$
\left[E_{n}+2 x\right] \beta_{n}^{\prime \prime}+3 \beta_{n}^{\prime}+d \beta_{n}=0
$$

where we have introduced notation :

$$
E_{n}=\frac{\left[B_{n}+B^{0}\right]^{2}}{\mu_{0} \alpha}, d=-\frac{1 \partial \alpha}{c \partial T} .
$$

Solution of equation (7) may be written in following form :

$$
\beta_{n}(x)=\frac{C_{1 n} \cos \sqrt{d\left(E_{n}+2 x\right)}+C_{2 n} \sin \sqrt{d\left(E_{n}+2 x\right)}}{\sqrt{E_{n}+2 x}} .
$$

The successive stability limits fields we determine from an equation (9) and additional boundary conditions : $\beta_{n}(0)=0,-\beta_{n}\left(x_{n-1}\right)<0$ and after substituting equation (9) to constitutive equation (5) :

$$
\begin{aligned}
& C_{1 n} \cos r\left(B_{n}+B^{0}\right)+C_{2 n} \sin r\left(B_{n}+B^{0}\right)=0 \\
& C_{1 n} \cos r\left(B_{n-1}^{+}+B^{0}\right)+C_{2 n} \sin r\left(B_{n-1}^{+}+B^{0}\right)=W_{n}
\end{aligned}
$$

$C_{1 n} \sin r\left(B_{n-1}^{+}+B^{0}\right)-C_{2 n} \cos r\left(B_{n-1}^{+}+B^{0}\right)=0$

where we have introduced the notation :

$$
W_{n}=\frac{\beta_{n}\left(x_{n-1}\right)\left(B^{0}+B_{n-1}^{+}\right)}{\sqrt{\mu_{0} \alpha}}, \quad r=\sqrt{\frac{d}{\mu_{0} \alpha}} .
$$

In equations (10-12) appear values of $n$-th stability limit field $B_{n}$ and $(n-1)$-th flux jump field, because boundary conditions determine just these values. From equations (10-12) we can directly find recurrence relation determining successive stability limit fields. At this aim we notice firstly that from equations (1112) follows normalizing condition on the constants $C_{1 n}$ and $C_{2 n}$ :

$C_{1 n}^{2}+C_{2 n}^{2}=W_{n}^{2}$ and $C_{1 n}=W_{n} \cos r\left(B_{n-1}^{+}+B^{0}\right)$.

We join next equations (10-12) and use result (14) what brings final dependence describing successive stability limit field $B_{n}$ in the function of previous flux jump field $B_{n-1}^{+}$:

$$
\cos r\left(B_{n}-B_{n-1}^{+}\right)=0 .
$$

First solution of equation (15) gives final relation :

$$
B_{n}=B_{n-1}^{+}-B^{1}
$$

where $B^{1}=\pi / 2 r$ is the first stability limit for increasing magnetic field.

For simultaneously solving recurrence equation (16) we should else determine the relation joining $n$-th stability limit field $B_{n}$ with corresponding flux jump field value $B_{n}^{+}$. As it has been indicated in [2] we can propose two simple relations between stability limit field and flux jump field : 1) if all instabilities develop into flux jumps and 2) assume that flux jump field is proportional to limited stability field. In first case has the place an equality : $B_{n}^{+}=B_{n}$, while second one is described with the condition : $B_{n}^{+}=q B_{n}$, where coefficient of proportionality $q$ for the case of decreasing magnetic field is smaller than unity. 
For mentioned above cases we find two families of flux jumps positions determined by equation (16). In the first one we receive :

$$
B_{n}^{+}=B_{1}-(n-1) B^{1}
$$

where $B_{1}$ is first stability limit in decreasing magnetic field, value of which has been determined in [3] :

$$
B_{1}=-B^{0}-2 B^{1}+\sqrt{\left(B^{m}+B^{0}\right)^{2}+2\left(B^{1}\right)^{2}}
$$

$B_{\mathrm{m}}$ is maximal magnetic induction in the cycle. In second case however we write equation (16) in the form :

$$
B_{n}^{+}=q B_{n-1}^{+}-q B^{1} \quad n=2,3,4, \ldots,
$$

which can be described by corresponding sum of geometrical progress :

$$
B_{n}^{+}=q^{n} B_{1}-q B^{1} \frac{1-q^{n-1}}{1-q} .
$$

Results (17) and (20), second term of which gives $(n-1)$-th flux jump field for increasing from null external magnetic field indicate an important function of parameter $B^{m}$, connected with $B_{1}$ through equation (18), in determining flux jumps position. Subsequent flux jumps fields in first case are only shifted dependent on $B_{1}$ value, while in second one it is deviation from this law given by power function $q^{n}$.

Proportionality between stability limit field and flux jumps field, assumed in above considerations may be deduced from papers $[4,5]$, in which the specific heat has been varied by filling porous $\mathrm{Nb}_{3} \mathrm{Sn}$ with liquid helium. Then specific heat per unit volume had also the contribution from heat vaporization of liquid. For isothermal conditions this can increase $c$ value 100 time, what really increases observed flux jumps field tenfold. Such dependence is in agreement with relation describing first stability limit field, what supports the assumption of proportionality between stability limit and flux jumps field. In particular case the coefficient of proportionality $q$ is equal to unity, what gives flux jumps position described with an equation (17).

We should remember however that flux jumps, positions of which are determined with equations (17) and (20) will appear if full stability field is not reached before, what according to [1] can be interpreted that magnetic field does not penetrate into the centre of superconductor. To determine value of full stability field we firstly consider, in which way varies magnetic flux penetration depth for $n$-th flux jump in the function of $n-x_{n-1}$. At this aim applying equation (6) we describe $x_{n-1}$ in the form :

$$
2 \mu_{0} \alpha x_{n-1}=\left(B_{n-1}^{+}-B_{n}^{+}\right)\left(2 B^{0}+B_{n-1}^{+}+B_{n}^{+}\right)
$$

which leads in both described cases to the relation $\partial x_{n-1} / \partial n<0$, where we have treated $n$ as a continuous variable. This result means that :

$$
x_{1}>x_{2}>x_{3}>x_{4} \ldots
$$

It remains however the question if $x_{0}>x_{1}$ ? For first case applying result of paper [3] and equation (18) we receive positive answer on this subject, while for flux jumps described with geometrical progress result depends on the values of phenomenological parameters : $q, B^{1}, B_{1}, B^{0}$. It means that in first case full stability limit will be reached either for first flux jump or in decreasing magnetic field appear many successive flux jumps, number of which is determined by the relation :

$$
n<1+B_{1} / B^{1} .
$$

In second case maximal number of flux jumps in decreasing magnetic field is limited by following inequality :

$$
n<1-\frac{\ln \left[1+(1-q) B_{1} / B^{1}\right]}{\ln q} .
$$

This number of flux jumps will appear however if full stability limit will be not reached for first or second flux jump. Above result indicates that in second case, in principle, may rise phenomenon that appear first flux jump in decreasing magnetic field, while further disappear. In first case however sample is internally stable or appear many jumps. It should be interesting to verify this subject experimentally.

In above calculations it has been assumed in fact isothermal flux density profile, it means that after thermal dissipation in the flux jumps process sample will be effectively cooled by liquid helium bath and before subsequent instability process recovers flux density profile specific for given bath temperature Such assumption in fact seems to be fulfilled in various experimental papers, for example in excellent paper of Coffey [4], who observed five flux jumps on $\mathrm{Nb}_{40} \mathrm{Ti}_{60}$ sample in field up to $4 \mathrm{~T}$. During the measurements after flux jump appearance, magnetic field was kept on constant level and next varied slowly with sweep rate $300 \mathrm{G} / \mathrm{min}$.

The time required for thermal recovery of superconducting material can be roughly estimated on the basis of paper [1], in which it was calculated temperature distribution function $f$ in the penetration layer for increasing time parameter $\theta=\alpha_{\mathrm{th}} t / x_{n}^{2}, x_{n}$ is defined earlier thickness of penetration layer, $t$ - time, while $\alpha_{\mathrm{th}}=K / c$ thermal diffusivity. If we take the values of specific heat per unit volume $\mathrm{NbTi}$ as equal to $2.5 \mathrm{~mJ} / \mathrm{cm}^{3} . \mathrm{K}$ according to [5] and $K$ heat conductivity as equal to $1 \mathrm{~mW} / \mathrm{cm} . \mathrm{K}$ on the basis of [6], while thickness of magnetic flux penetration $\sim 0.3 \mathrm{~cm}$, we receive then the value of time parameter $\theta=1$ for $t=0.25 \mathrm{~s}$. Function $f$ describes the steadness of temperature distribution and for $\theta=1$ is smaller 
than 0.1 , what is rather near to steady state value, when $f=0$, and temperature in all points of sample is equal to helium bath temperature.

Although these considerations were performed for simplified assumption $\mathrm{d} Q / \mathrm{d} t=$ const., they probably allow to estimate the temperature distribution in hard superconductors. In NbTi for example, as it can be deduced from Coffey work [4], the distance between flux jumps is about $0.8 \mathrm{~T}$, it means that for magnetic field sweep rate $0.2 \mathrm{~T} / \mathrm{min}$ the time in which subsequent flux jumps will appear is about three ranges higher than previously estimated thermal recovery time. It supports applied in this paper assumption of describing subsequent instability process with an isothermal magnetic field profile distribution.

\section{Conclusions.}

The results of calculations successive flux jumps fields positions in decreasing magnetic field for pinning forces described with Kim's critical state model are described. Full set of flux jumps positions for two simple physical cases has been given, while differences in comparison with results received for increasing magnetic field discussed.

\section{References}

[1] Wipf, S. L., Phys. Rev. 161 (1967) 404.

[2] Sosnowski, J., Phys. Status Solidi (a) 79 (1983) 207.

[3] Sosnowski, J., LT-17 Contributed Papers, p. 1075, Eds. W. Eckern, A. Schmid, W. Weber, H. Wühl (Elsevier Science Publishers B.V.) 1984.

[4] Coffey, H. T., Cryogenics 7 (1967) 73.

[5] BReChNA, H., Superconducting Magnet Systems (Springer-Verlag, Berlin) 1973.

[6] Kwasnitza, K., Barbisch, B., Hulliger, F., Cryogenics 23 (1983) 649. 